\title{
QUENCHING SOLUTIONS OF A STOCHASTIC PARABOLIC PROBLEM ARISING IN ELECTROSTATIC MEMS CONTROL
}

\author{
NIKOS I. KAVALLARIS
}

\begin{abstract}
In the current paper, we consider a stochastic parabolic equation which actually serves as a mathematical model describing the operation of an electrostatic actuated micro-electro-mechanical system (MEMS). We first present the derivation of the mathematical model. Then after establishing the local well-posedeness of the problem we investigate under which circumstances a finite-time quenching for this SPDE, corresponding to the mechanical phenomenon of touching down, occurs. For that purpose the Kaplan's eigenfunction method adapted in the context of SPDES is employed.
\end{abstract}

\section{INTRODUCTION}

In the current work we consider the following stochastic semilinear parabolic problem

$$
\begin{aligned}
& u_{t}=\Delta u+\frac{\lambda}{(1-u)^{2}}+\sigma(u) \partial_{t} W(x, t), \quad \text { in } \quad Q_{T}:=\Omega \times(0, T), T>0, \\
& u=0, \quad \text { on } \Gamma_{T}:=\partial \Omega \times(0, T), \\
& u(x, 0)=\xi(x), \quad x \in \Omega,
\end{aligned}
$$

where $\Omega$ is a bounded subset of $\mathbb{R}^{N}, N=1,2,3$, with smooth boundary and $\lambda$ is a positive parameter. Here $\partial_{t} W(x, t)$ denotes by convention the formal (time) derivative of the Wiener random process $W(x, t)$ in a complete probability space $\left(\mathcal{B}, \mathcal{F}_{t}, \mathbb{P}\right)$ with filtration $\left(\mathcal{F}_{t}\right)_{t \in[0, T]}$ which is defined more rigorously in the Section 3. Then $\sigma(u) \partial_{t} W$ is a multiplicative noise and $\sigma(u)$ is actually the magnitude of the noise term and its growth will be identified later. Furthermore, the initial data $\xi: \mathcal{B} \times \Omega \rightarrow \mathbb{R}^{+}$are considered to be almost surely (a.s.) positive. Then the solution $u=u(x, t ; \omega)$ of $(1.1)-(1.3)$, for any $\omega \in \mathcal{B}$, is a stochastic process and the investigation of its basic properties is the main purpose of the current work.

In case $\sigma(u) \equiv 0$ then problem (1.1)-(1.3) is reduced to its deterministic counterpart

$$
\begin{aligned}
& u_{t}=\Delta u+\frac{\lambda}{(1-u)^{2}}, \quad \text { in } \quad Q_{T}, \\
& u=0 \quad \text { on } \Gamma_{T}, \\
& 0 \leq u(x, 0)=\xi(x)<1, \quad x \in \Omega .
\end{aligned}
$$

Actually (1.4)-(1.6) and its non-local variations have attracted the attention of many researchers $[6,7,10,9,11,13,16,17]$, since this kind of equations can model the operation of some (idealized) electrostatic actuated micro-electro-mechanical systems (MEMS) which have a wide variety of applications.

Date: August 26, 2016.

1991 Mathematics Subject Classification. Primary 60H15, 35B44 ; Secondary 34B10, 35B50, 35B51.

Key words and phrases. Electrostatic MEMS, touchdown, quenching, stochastic semilinear partial differential equations. 
The associated stationary problem to (1.4)-(1.6) is

$$
\begin{aligned}
& \Delta w+\frac{\lambda}{(1-w)^{2}}=0, \quad \text { in } \Omega, \\
& w=0 \text { on } \partial \Omega,
\end{aligned}
$$

and we define

$$
\lambda^{*}:=\sup \{\lambda>0: \text { problem (1.7)-(1.8) has classical solution corresponding to } \lambda\} \text {, }
$$

where $0<\lambda^{*}<\infty$ for any $d=1,2,3$, see $[6,8,14]$.

Regarding the dynamics of (1.4)-(1.6), whenever $0<\lambda<\lambda^{*}$ and for "small enough" initial data $\xi$ then the solution $u$ of (1.4)-(1.6) converges to a steady-state solution, $[6,9,16]$. On the other hand, either for initial data $\xi(x)$ "close enough" to 1 and $0<\lambda<\lambda^{*}$, or for any initial data $0<\xi(x)<1$ and $\lambda>\lambda^{*}$ then a finite-time quenching occurs, i.e. there exists $T_{q}<\infty$ such that $\|u(\cdot, t)\|_{\infty} \rightarrow 1$ as $t \rightarrow T_{q}$. Finally, when there is a weak steadystate $w^{*}$ with $\left\|w^{*}\right\|_{\infty}=1$ at $\lambda^{*}$ then a infinite-time quenching takes place. Obviously, quenching yields a singular behaviour of the equation (1.4) since its reaction term becomes unbounded close to the quenching time $T_{q}$, but it is also linked with a possible destruction of the MEMS device, [21]. The destruction might be a consequence of the touching down of an elastic electrode towards its opposite rigid one within the MEMS device, see Figure 1 below. One of the aims of the current work is to investigate whether there is an impact of the noise term on the occurrence of finite-time quenching.

The structure of the paper is as follows. In Section 2 a brief derivation of the mathematical model (1.1)-(1.3) is presented. In section 3 we provide the preliminary mathematical tools will need throughout the manuscript. Section 4 is devoted to the establishment of the local well-posedeness of problem (1.1)-(1.3). Finally Section 5 investigates the finite-time quenching for the solution $u$ of (1.1)-(1.3).

\section{The Mathematical Model}

The motivation for studying problem (1.1)-(1.3) is its association with the operation of some electrostatic actuated MEMS. The term "MEMS" more precisely refers to precision devices which combine mechanical processes with electrical circuits. In particular, electrostatic actuation is a popular application of MEMS. MEMS devices range in size from millimetres down to microns, and involve precision mechanical components that can be constructed using semiconductor manufacturing technologies. Various electrostatic actuated MEMS have been developed and used in a wide variety of devices applied as sensors and have fluid-mechanical, optical, radio frequency $(\mathrm{RF})$, data-storage, and biotechnology applications. Examples of microdevices of this kind include microphones, temperature sensors, RF switches, resonators, accelerometers, micromirrors, micropumps, microvalves, data-storage devices etc., [20, 21].

The key part of such a electrostatic actuated MEMS device usually includes an elastic plate (or membrane) suspended above a rigid ground one. Typically the elastic plate is held fixed at two ends while the other two edges remain free to move, see Figure 1. Once a potential difference $V$ is applied between the elastic membrane and the rigid ground plate, the membrane starts deflecting towards it. Consider now the situation when the width $d$ of the gap, between the membrane and the bottom plate, is small compared to the device length $L$, then the deformation of the elastic membrane $u$, after proper scaling, can 


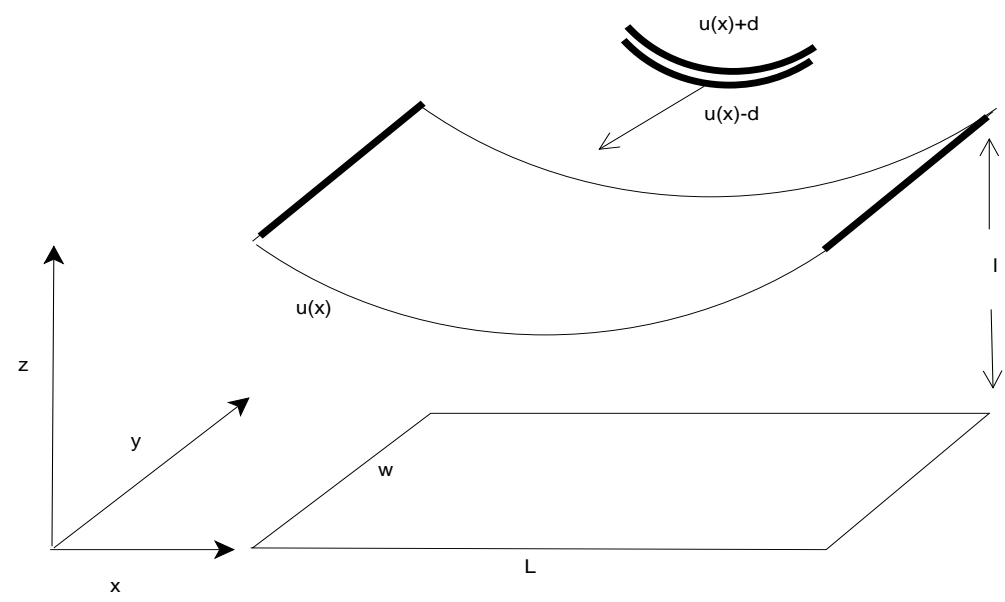

FIGURE 1. Sketch of the capacitive control circuit (controlled-voltage operation).

described by the dimensionless equation

$$
u_{t}=\Delta u+\frac{\lambda g(x, t)}{(1-u)^{2}}, \quad x \in \Omega, t>0,
$$

see $[19,20]$. The function $g(x, t)$ describes the varying dielectric properties of the membrane and for some elastic materials can be taken to be constant; for simplicity we assume here that $g(x, t) \equiv 1$.

Furthermore, the parameter $\lambda$ in (2.1) equals to

$$
\lambda=\frac{V^{2} L^{2} \varepsilon_{0}}{2 \mathcal{T} \ell^{2}}
$$

and actually tunes the whole process. Here $\mathcal{T}$ stands for the tension of the elastic membrane, while $\ell$ is the characteristic width of the gap between the membrane and the fixed ground plate (electrode), and $\varepsilon_{0}$ is the permittivity of free space. It has been experimentally observed, see [21], that there is a significant uncertainty regarding the values of $V$ and $\mathcal{T}$. In particular, $V$ fluctuates around an average value $V_{0}$, hence this implies that $\lambda=\lambda_{0}+\alpha \eta(x, t)$ where $\alpha>0$ is a coefficient measuring the intensity of the noise term $\eta(x, t)$. The coefficient $\alpha$ might depend on the deformation $u$ as well, whereas the noise $\eta(x, t)$ could be taken to be a space-time white noise i.e. $\eta(x, t)=\partial_{t} W(x, t)$, where $W(x, t)$ is a Wiener process. Therefore (2.1) is finally transformed to (1.1).

Since, the two edges of the membrane are kept fixed then Dirichlet boundary conditions of the form (1.2) are imposed together initial conditions $u(x, 0)=\xi(x)$.

The mathematical model (1.1)-(1.3) as a stochastic perturbation of (1.4)-(1.6), captures possible destructions due to the environment of the MEMS system and hence, under some circumstances, is more realistic. To the best of our knowledge this problem has not been considered before in the literature of SPDEs, despite its mathematical importance. Indeed, its mathematical interest arises from the presence of singular term $f(u)=1 /(1-u)^{2}$ which leads to the occurrence of the mathematical phenomenon of finite-time quenching closely associated with the mechanical phenomenon of touching down. Therefore, it is reasonable for someone to investigate whether (1.1)-(1.3) can predict the occurrence of finite-time 
quenching as it happens with the deterministic problem (1.4)-(1.6). The main purpose of the current paper is to provide a response to this question. We affirmatively answer this question in Section 5.

\section{Preliminaries}

In this section we introduce the main mathematical concepts we will need throughout the text. In the following, $C, K$ will denote positive constants whose values might change from line to line.

A $Q$-Wiener process $\{W(\cdot, t): t \geq 0\}$ is defined as a $H$-valued process, where $Q \in L(H)$ is non-negative definite and symmetric and has an orthonormal basis $\chi_{j}(x) \in H, j=$ $1,2,3, \ldots$ of eigenfunctions with corresponding eigenvalues $\gamma_{j} \geq 0, j=1,2,3, \ldots$ such that $\operatorname{Tr}(Q)=\sum_{j=1}^{\infty} \gamma_{j}<\infty$. (i.e., $Q$ is of trace class). It is well-known, see [18], that $W(\cdot, t)$ is a $Q$-Wiener process if and only if

$$
W(x, t)=\sum_{j=1}^{\infty} \gamma_{j}^{1 / 2} \chi_{j}(x) \beta_{j}(t), \quad \text { almost surely (a.s.) },
$$

where $\beta_{j}(t)$ are independent and identically distributed $\mathcal{F}_{t}$-Brownian motions and the series converges in $L^{2}(\mathcal{B}, H)$. We point out that the eigenfunctions $\left\{\chi_{j}(x)\right\}_{j=1}^{\infty}$ may be different from the eigenfunctions $\left\{\phi_{j}(x)\right\}_{j=1}^{\infty}$ of the elliptic operator $A=-\Delta: \mathcal{D}(A)=H^{2}(\Omega) \cap$ $H_{0}^{1}(\Omega) \subset H \rightarrow H$, which is self-adjoint, positive definite with compact inverse. Note that the trace class operator $Q$ is also a Hilbert -Schmidt operator.

For each Hilbert-Schmidt operator $Q$ on $H=L^{2}(\Omega)$, there exists a kernel $q(x, y)$ such that, $[2,18]$

$$
(Q u)(x):=\int_{\Omega} q(x, y) u(y) d y, \quad \text { for any } x \in \Omega, u \in H,
$$

and

$$
\|Q\|_{H S(H, H)}=\|q\|_{L^{2}(\Omega \times \Omega)} .
$$

The kernel $q(x, y)$ is also called the covariance function of the $Q$-Wiener process $W(\cdot, t)$ and $\|\cdot\|_{H S}$ denotes the Hilbert-Schmidt norm.

Let $U_{0}=:\left\{Q^{1 / 2} u: u \in H\right\}$ then we define $\mathcal{L}_{0}^{2}$ to be the set of linear operators $\mathcal{J}: H \rightarrow H$ equipped with the norm

$$
\|\mathcal{J}\|_{\mathcal{L}_{0}^{2}}^{2}:=\left(\sum_{j=1}^{\infty}\left\|\mathcal{J} Q^{1 / 2} \chi_{j}\right\|_{2}\right)=\left\|\mathcal{J} Q^{1 / 2}\right\|_{H S(H, H)}^{2}<\infty .
$$

Furthermore we denote by $L^{2}(\mathcal{B}, H)$ the space of all random variables $X: \mathcal{B} \rightarrow H$ equipped with the norm

$$
\|X(\omega)\|_{L^{2}(\mathcal{B}, H)}:=\mathbb{E}\left[\|X(\omega)\|_{H}^{2}\right]^{1 / 2}<\infty, \quad \text { for any } \omega \in \mathcal{B},
$$

known also as the space of the mean-square integrable random variables, where $\mathbb{E}$ stands for the expectation in the probability space $\left(\mathcal{B}, \mathcal{F}_{t}, \mathbb{P}\right)$.

We note that for the above Wiener process there holds,

$$
\mathbb{E}[W]=0 .
$$

It is easily seen that $f: H \rightarrow H$, defined as $f(u)=\frac{1}{(1-u)^{2}}$, satisfies a local Lipschitz condition, i.e. for any $0<\rho<1$ and $u_{1}, u_{2} \in B_{\rho}:=\left\{u \in L^{\infty}(\Omega):\|u\|_{\infty}<\rho\right\}$ there exists 
$C_{\rho}>0$ such that

$$
\left\|f\left(u_{1}\right)-f\left(u_{2}\right)\right\|_{H} \leq C_{\rho}\left\|u_{1}-u_{2}\right\|_{H} .
$$

An immediate consequence of (3.3) is the following growth condition

$$
\| f(u)) \|_{H} \leq C_{\rho}\left(1+\|u\|_{H}\right) \quad \text { for any } \quad u \in B_{\rho} .
$$

Now, we assume that there exists a Lipschitz continuous function $b: \mathbb{R} \rightarrow \mathbb{R}$ such that

$$
\sigma: H \rightarrow \mathcal{L}_{0}^{2} \quad \text { with } \quad(\sigma(u) \psi)(x):=b(u(x)) \psi(x) \text { for any } u, \psi \in H,
$$

then $\sigma$ satisfies a local Lipschitz condition and a linear growth condition as well ([18, Lemma 10.24]), in particular for any $0<\rho<1$ there exists $K_{\rho}>0$ such that for any $u_{1}, u_{2}, u \in B_{\rho}$,

$$
\left\|\sigma\left(u_{1}\right)-\sigma\left(u_{2}\right)\right\|_{\mathcal{L}_{0}^{2}} \leq K_{\rho}\left\|u_{1}-u_{2}\right\|_{H} \quad \text { and } \quad\|\sigma(u)\|_{\mathcal{L}_{0}^{2}} \leq K_{\rho}\left(1+\|u\|_{H}\right) .
$$

\section{Local Existence}

Problem (1.1)-(1.3) can be considered as an Itô equation in the Hilbert space $H$ and is rewritten by suppressing the dependence on space as

$$
\begin{aligned}
& d u_{t}=\left[-A u_{t}+\lambda f\left(u_{t}\right)\right] d t+\sigma\left(u_{t}\right) d W_{t}, \quad 0<t<T, \\
& u_{0}=\xi
\end{aligned}
$$

where $u_{t}=u(\cdot, t)$ can be interpreted as a predictable $H$-valued stochastic process recalling that $A=-\Delta: \mathcal{D}(A)=H^{2}(\Omega) \cap H_{0}^{1}(\Omega) \subset H \rightarrow H$. Moreover, $-A$ is the generator of an analytic semigroup $E(t)=e^{-t A}$ on $H$.

We now introduce the following notions of solutions (4.1)-(4.2) will be used throughout the current manuscript, see also $[3,5,18]$.

Definition 4.1. A predictable $H$-valued stochastic process $\left\{u_{t}: t \in[0, T]\right\}$ is called a strong solution of (4.1)-(4.2) if $u_{t} \in \mathcal{D}(A)$ for any $t \in[0, T]$, with $\mathbb{P}\left[\sup _{t \in[0, T]}\left\|u_{t}\right\|_{\infty}<1\right]=1$, and

$$
u_{t}=\xi+\int_{0}^{t}\left[-A u_{s}+\lambda f\left(u_{s}\right)\right] d s+\int_{0}^{t} \sigma\left(u_{s}\right) d W_{s}, \quad \mathbb{P}-\text { a.s. },
$$

where the last integral in (4.3) is a stochastic integral which is well defined, see Theorem 2.4 in $[2]$.

Definition 4.2. A predictable $H$-valued stochastic process $\left\{u_{t}: t \in[0, T]\right\}$ such that $\mathbb{P}\left[\sup _{t \in[0, T]}\left\|u_{t}\right\|_{\infty}<1\right]=1$ is called a weak solution of (4.1)-(4.2) if for any $v \in \mathcal{D}(A), t \in$ $[0, T]$

$$
\left(u_{t}, v\right)=(\xi, v)+\int_{0}^{t}\left[-\left(u_{s}, A v\right)+\left(\lambda f\left(u_{s}\right), v\right)\right] d s+\int_{0}^{t}\left(\sigma\left(u_{s}\right) d W_{s}, v\right), \quad \mathbb{P}-a . s .
$$

where $(\cdot, \cdot)$ stands for the inner product into $H$.

Definition 4.3. A predictable $H$-valued stochastic process $\left\{u_{t}: t \in[0, T]\right\}$ such that $\mathbb{P}\left[\sup _{t \in[0, T]}\left\|u_{t}\right\|_{\infty}<1\right]=1$ is called a mild solution of (4.1)-(4.2) if for any $t \in[0, T]$, there holds

$$
u_{t}=E(t) \xi+\lambda \int_{0}^{t} E(t-s) f\left(u_{s}\right) d s+\int_{0}^{t} E(t-s) \sigma\left(u_{s}\right) d W_{s}, \quad \mathbb{P} \text {-a.s. and x-a.e. in } \Omega \text {. }
$$


Now the weak (variational) solution is equivalent to the mild solution under the assumption of the local Lipschitz continuity of $f$, see [12]. The positivity of solution of (1.1)-(1.3) (or equivalently of (4.1)-(4.2)) follows easily from comparison arguments (see e.g. [1] or $[4])$.

Theorem 4.4. (Local Existence \& Uniqueness) Suppose that $\sigma$ satisfies (3.5). Fix $0<$ $r_{0}<1$ and consider initial data $\xi \in L^{2}(\mathcal{B}, \mathcal{D}(A))$ such that $\|\xi\|_{L^{2}(\mathcal{B}, \mathcal{D}(A))}<r_{0}$, then there exists $T=T\left(r_{0}\right)>0$ such that problem (4.1)-(4.2) admits a unique mild solution $u_{t}$ in $[0, T]$.

Furthermore, there exists $C_{T}>0$ such that

$$
\sup _{0 \leq t \leq T}\left\|u_{t}\right\|_{L^{2}(\mathcal{B}, \mathcal{D}(A))} \leq C_{T}\left(1+\|\xi\|_{L^{2}(\mathcal{B}, \mathcal{D}(A))}\right)
$$

Proof. Denote by $\mathcal{S}_{T}$ the Banach space of $H$-valued predictable processes $\left\{u_{t}: t \in[0, T]\right\}$ equipped with the norm

$$
\left\|u_{t}\right\|_{\mathcal{S}_{T}}:=\sup _{0 \leq t \leq T}\left\|u_{t}\right\|_{L^{2}(\mathcal{B}, H)}
$$

Now for any $0<r_{0}<\rho<1$ set

$$
\mathcal{S}_{\rho, T}:=\left\{u_{t} \in \mathcal{S}_{T}:\left\|u_{t}\right\|_{\mathcal{S}_{\rho, T}}:=\sup _{0 \leq t \leq T}\left\|u_{t}\right\|_{L^{2}(\mathcal{B}, \mathcal{D}(A))} \leq \rho<1\right\}
$$

For any $u_{t} \in \mathcal{S}_{\rho, T}$ we define

$$
\mathcal{M}\left(u_{t}\right):=E(t) \xi+\lambda \int_{0}^{t} E(t-s) f\left(u_{s}\right) d s+\int_{0}^{t} E(t-s) \sigma\left(u_{s}\right) d W_{s} .
$$

Our purpose is to employ Banach's fixed point theorem to prove existence and uniqueness of the equation $\mathcal{M}\left(u_{t}\right)=u_{t}$ in $\mathcal{S}_{\rho, T}$.

Step 1: We first show that $\mathcal{M}$ maps $\mathcal{S}_{\rho, T}$ into itself. We note that $\mathcal{M}\left(u_{t}\right)$ is a $H$-valued predictable process since $\xi$ is $\mathcal{F}_{0}$-measurable and the stochastic integral is a predictable process. Therefore it suffices to prove that $\left\|\mathcal{M}\left(u_{t}\right)\right\|_{\mathcal{S}_{\rho, T}}<\rho$.

We first note that

$$
\|E(t) \xi\|_{\mathcal{S}_{1, T}} \leq\|\xi\|_{\mathcal{S}_{\rho, T}}<r_{0}
$$

by our hypothesis.

Note that if $u_{t} \in \mathcal{S}_{\rho, T}$ then by virtue of Sobolev's inequality we can find $\rho_{0}$ small enough such that

$$
\mathbb{E}\left[\left\|u_{t}\right\|_{\infty}\right] \leq \rho_{0}<1
$$

and hence (3.4) and (3.5) are satisfied. 
Next by using the growth condition (3.4) we also have

$$
\begin{aligned}
\left\|\int_{0}^{t} E(t-s) f\left(u_{s}\right) d s\right\|_{\mathcal{S}_{\rho, T}} & \leq \int_{0}^{t}\left\|E(t-s) f\left(u_{s}\right)\right\|_{\mathcal{S}_{\rho, T}} d s \\
& \leq \int_{0}^{t}\left\|f\left(u_{s}\right)\right\|_{\mathcal{S}_{\rho, T}} d s \\
& \leq \int_{0}^{t} C_{\rho_{0}}\left(1+\left\|u_{s}\right\|_{\mathcal{S}_{\rho, T}}\right) d s \\
& \leq C_{\rho_{0}} T\left(1+\sup _{0 \leq s \leq T}\left\|u_{s}\right\|_{\mathcal{S}_{\rho, T}}\right) \\
& \leq 2 C_{\rho_{0}} T,
\end{aligned}
$$

for any $0<t<T$.

Furthermore by virtue of Itô's isometry (see page 322 in [18]) and (3.5) we have for any $0<t<T$

$$
\begin{aligned}
\left\|\int_{0}^{t} E(t-s) \sigma\left(u_{s}\right) d W_{s}\right\|_{\mathcal{S}_{\rho, T}}^{2} & =\int_{0}^{t} \mathbb{E}\left[\left\|E(t-s) \sigma\left(u_{s}\right)\right\|_{\mathcal{L}_{0}^{2}}^{2}\right] d s \\
& \leq K_{\rho_{0}}^{2} \int_{0}^{t}\|E(t-s)\|_{\mathcal{L}(H)}^{2} d s\left(1+\sup _{0 \leq s \leq t}\left\|u_{s}\right\|_{\mathcal{S}_{\rho, T}}\right)^{2},
\end{aligned}
$$

where $\mathcal{L}(H)$ is the set of all linear operators from $H$ into $H$.

Using a well known semigroup estimate, see for example page 480 in [18],

$$
\int_{0}^{T}\|E(t)\|_{\mathcal{L}(H)}^{2} d t \leq K^{2} T
$$

where $K$ is a positive constant, we finally derive

$$
\left\|\int_{0}^{t} E(t-s) \sigma\left(u_{s}\right) d W_{s}\right\|_{\mathcal{S}_{1, T}} \leq 2 K_{1} T^{1 / 2}, \quad 0<t<T,
$$

for some $K_{1}>0$.

Combining now (4.8)-(4.12) we obtain that

$$
\begin{aligned}
\left\|\mathcal{M}\left(u_{t}\right)\right\|_{\mathcal{S}_{\rho, T}} & <r_{0}+2 \lambda C_{\rho_{0}} T+2 K_{1} T^{1 / 2} \\
& \leq r_{0}+2\left(\lambda C_{\rho_{0}} T+K_{1} T^{1 / 2}\right) \\
& \leq \rho,
\end{aligned}
$$

by choosing $T$ small enough, say smaller than some $T_{1}=T_{1}\left(\lambda, \rho, r_{0}\right)$, such that

$$
2\left(\lambda C_{\rho_{0}} T+K_{1} T^{1 / 2}\right)<\rho-r_{0}, \quad \text { for any } \quad 0<T<T_{1} .
$$

Step 2: We now show that the operator $\mathcal{M}$ is a contraction, i.e. there is a positive constant $0<\gamma<1$ such that

$$
\left\|\mathcal{M}\left(u_{t}\right)-\mathcal{M}\left(v_{t}\right)\right\|_{\mathcal{S}_{\rho, T}} \leq \gamma\left\|u_{t}-v_{t}\right\|_{\mathcal{S}_{\rho, T}} .
$$


In fact, we have

$$
\begin{aligned}
\mathcal{M}\left(u_{t}\right)-\mathcal{M}\left(v_{t}\right)= & \lambda \int_{0}^{t} E(t-s)\left(f\left(u_{s}\right)-f\left(v_{s}\right)\right) d s \\
& +\int_{0}^{t} E(t-s)\left(\sigma\left(u_{s}\right)-\sigma\left(v_{s}\right)\right) d W_{s} .
\end{aligned}
$$

Then by virtue of (3.3) and (3.5) and using Itô's isometry together with (4.11) we derive

$$
\left\|\mathcal{M}\left(u_{t}\right)-\mathcal{M}\left(v_{t}\right)\right\|_{\mathcal{S}_{\rho, T}}^{2} \leq 4 \lambda^{2} \int_{0}^{t} C_{\rho_{0}}^{2}\left\|u_{s}-v_{s}\right\|_{\mathcal{S}_{\rho, T}}^{2} d s+2 C T\left\|u_{t}-v_{t}\right\|_{\mathcal{S}_{\rho, T}}^{2}
$$

for $\rho_{0}$ small enough and $C>0$ and finally

$$
\left\|\mathcal{M}\left(u_{t}\right)-\mathcal{M}\left(v_{t}\right)\right\|_{\mathcal{S}_{\rho, T}}^{2} \leq\left(4 \lambda^{2} C_{\rho_{0}}^{2}+2 C\right) T\left\|u_{t}-v_{t}\right\|_{\mathcal{S}_{\rho, T}}^{2}
$$

which implies that $\mathcal{M}$ is a contraction on $\mathcal{S}_{\rho, T}$ if

$$
T<T_{2}:=\frac{1}{\left(4 \lambda^{2} C_{\rho_{0}}^{2}+2 C\right)} .
$$

Therefore by choosing $T_{0}=\min \left\{T_{1}, T_{2}\right\}$ we obtain by Banach's fixed point theorem that $\mathcal{M}$ has a unique fixed point in $\mathcal{S}_{\rho, T}$ for $0<T<T_{0}$ and thus (4.1)-(4.2) admits a unique mild solution in $\left[0, T_{0}\right]$.

Finally, bound (4.6) is immediately derived by a direct application of Gronwall's inequality.

Remark 4.5. Now according to the comments after Definition 4.3 the solution obtained by Theorem 4.4 is a weak solution which due to (4.9) satisfies $\mathbb{P}\left[\sup _{t \in[0, T]}\left\|u_{t}\right\|_{\infty}<1\right]=1$, and hence it is a local in time strong solution, see also [3, 5]. Furthermore, it is obvious by the proof of Theorem 4.4 the solution can be extended in time as long as $\left\|u_{t}\right\|_{\mathcal{S}_{\rho, T}}<1$ and only ceases to exist once $\left\|u_{t}\right\|_{\mathcal{S}_{\rho, T}}=1$, see next section.

\section{Finite-Time Quenching}

In this section we prove that the local-in-time solution of (1.1)-(1.3), under some circumstances, cannot be extended as a global-in-time solution and hence finite-time quenching takes place. To this end we employ a widely utilized classical technique of Kaplan ([15]), which essentially relies on testing (4.4) by positive Dirichlet eigenfunctions of the Laplacian. Before proceeding further to the proof of finite-time quenching we definite in the context of stochastic processes.

Definition 5.1. We say that the (strong) solution to the problem (1.1)-(1.3) quenches in finite time if $\mathbb{P}\left[T_{q}<\infty\right]=1$ where $T_{q}$ is the quenching time defined as $T_{q}=\inf \{t>0$ : $\left.\|u(\cdot, t)\|_{\mathcal{D}(A)}=1\right\}$.

Remark 5.2. Note that by virtue of Sobolev's inequality the violation of the inequality $\|u(\cdot, t)\|_{\mathcal{D}(A)}<1$ for $d=1,2,3$ may occur if there exists $T>0$ such that $\|u(\cdot, T)\|_{\infty}=1$.

Therefore in the current work we also use the following alternative definition which is more convenient for our approach.

Definition 5.3. The solution $u$ of problem (1.1)-(1.3) quenches in finite time if there exists $0 \leq T_{q}<\infty$ such that

$$
\limsup _{t \rightarrow T_{q}} \mathbb{E}\left[\|u(\cdot, t)\|_{\infty}\right]=1
$$


Our first quenching result is valid when the parameter $\lambda$ is large enough.

Theorem 5.4. Suppose that $\xi \in L^{2}(\mathcal{B} ; H)$ with $\mathbb{E}\left[\|\xi\|_{\infty}\right]<1$ then the solution $u$ of (1.1)(1.3) quenches in finite time for sufficiently large values of the parameter $\lambda$.

Proof. Let $\left(\lambda_{1}, \phi_{1}(x)\right)$ be the first eigenpair of the operator $A=-\Delta$, i.e. $\phi_{1}$ satisfies

$$
\begin{aligned}
& A \phi_{1}=\lambda_{1} \phi_{1}, x \in \Omega \\
& \phi_{1}=0, x \in \partial \Omega .
\end{aligned}
$$

Since it is well known that $\phi_{1}$ has a constant sign on $\Omega$ we can take $\phi_{1} \geq 0$ on $\bar{\Omega}$ and normalized such that

$$
\int_{\Omega} \phi_{1} d x=1
$$

Now putting $v=\phi_{1}$ into the weak formulation (4.4) we have

$$
\begin{aligned}
\hat{u}(t): \quad & =\int_{\Omega} u_{t} \phi_{1} d x \\
& =\int_{\Omega} \xi \phi_{1} d x-\int_{0}^{t} \int_{\Omega} u_{s} A \phi_{1} d x d s \\
& +\lambda \int_{0}^{t} \int_{\Omega} f\left(u_{s}\right) \phi_{1} d x d s+\int_{0}^{t} \int_{\Omega} \sigma\left(u_{s}\right) \phi_{1} d x d W_{s} \\
& =\int_{\Omega} \xi \phi_{1} d x-\lambda_{1} \int_{0}^{t} \int_{\Omega} u_{s} \phi_{1} d x d s \\
& +\lambda \int_{0}^{t} \int_{\Omega} f\left(u_{s}\right) \phi_{1} d x d s+\int_{0}^{t} \int_{\Omega} \sigma\left(u_{s}\right) \phi_{1} d x d W_{s} .
\end{aligned}
$$

Now taking the expectation over (5.2) we derive

$$
\begin{aligned}
\mathbb{E}[\hat{u}(t)] & =\mathbb{E}\left[\int_{\Omega} \xi \phi_{1} d x\right]-\lambda_{1} \mathbb{E}\left[\int_{0}^{t} \int_{\Omega} u \phi_{1} d x d s\right] \\
& +\lambda \mathbb{E}\left[\int_{0}^{t} \int_{\Omega} f\left(u_{s}\right) \phi_{1} d x d s\right] \\
& +\mathbb{E}\left[\int_{0}^{t} \int_{\Omega} \sigma(u) \phi_{1} d x d W_{s}\right]
\end{aligned}
$$

Note that

$$
\mathbb{E}\left[\int_{0}^{t} \int_{\Omega} \sigma(u) \phi_{1}(x) d x d W_{s}\right]=0,
$$

due to the fact that $W(x, t)$ is a Wiener process, see also (3.2).

We define $\Psi(t):=\mathbb{E}[\hat{u}(t)]$ then by virtue of (5.1)

$$
\Psi(t) \leq \mathbb{E}\left[\left\|u_{t}\right\|_{\infty} \int_{\Omega} \phi_{1} d x\right]=\mathbb{E}\left[\left\|u_{t}\right\|_{\infty}\right] .
$$

By interchanging the order of expectation and integration, by virtue of Fubini's theorerm, we deduce for $t \geq 0$,

$$
\Psi(t)=\Psi_{0}-\lambda_{1} \int_{0}^{t} \Psi(s) d s+\lambda \mathbb{E}\left[\int_{0}^{t} \int_{\Omega} f\left(u_{s}\right) \phi_{1} d x d s\right]
$$


where

or equivalently the differential form,

$$
\Psi_{0}=\mathbb{E}\left[\left(\xi, \phi_{1}\right)\right]=\mathbb{E}\left[\int_{\Omega} \xi \phi_{1} d x\right]
$$

$$
\frac{d \Psi}{d t}=-\lambda_{1} \Psi(t)+\lambda \mathbb{E}\left[\int_{\Omega} f\left(u_{s}\right) \phi_{1} d x\right], \quad t>0, \Psi(0)=\Psi_{0} .
$$

Now by virtue of Jensen's inequality, since $f(u)$ is a convex function, we deduce

$$
\begin{aligned}
\mathbb{E}\left[\int_{\Omega} f\left(u_{s}\right) \phi_{1} d x\right] & \geq \mathbb{E}\left[f\left(\int_{\Omega} u_{t} \phi_{1} d x\right)\right] \\
& =\mathbb{E}[f(\hat{u}(t))] \\
& \geq f(\Psi(t)) .
\end{aligned}
$$

Combining (5.5) with (5.6),

$$
\frac{d \Psi(t)}{d t} \geq-\lambda_{1} \Psi(t)+\lambda f(\Psi(t)), \quad t>0, \quad \Psi(0)=\Psi_{0}
$$

Now we compare $\Psi(t)$ with the solution $H(t)$ of the following initial value problem

$$
\frac{d H(t)}{d t}=-\lambda_{1} H(t)+\lambda f(H(t)), \quad t>0, \quad H(0)=\Psi_{0}
$$

to derive that $\Psi(t) \geq H(t)$ on their domain of existence.

Solving analytically (5.8) and putting $H=1$ as an upper limit of integration then the quenching time for $\widehat{T}$ for $H$ is given by

$$
\widehat{T}=\int_{\Psi_{0}}^{1} \frac{1}{\left(\lambda f(\tau)-\lambda_{1} \tau\right)} d \tau
$$

and is finite if the improper integral in (5.9) converges.

Recalling that $f(u)=1 /(1-u)^{2}$ it can be easily seen that $\widehat{T}$ is finite provided that

$$
\lambda>\widehat{\lambda}:=\frac{4 \lambda_{1}}{27} .
$$

Finally (5.4) implies that the quenching time $T_{q}$ of (1.1)-(1.3) must be also finite. In particular, for any $\lambda>\widehat{\lambda}$ there holds

$$
T_{q} \leq \widehat{T}=\int_{\Psi_{0}}^{1} \frac{1}{\left(\lambda f(\tau)-\lambda_{1} \tau\right)} d \tau
$$

This completes the proof of the Theorem.

Finite-time quenching occurs for "large enough" initial data which are close enough to 1 as well. Indeed the following result is valid.

Theorem 5.5. Suppose that $\xi \in L^{2}(\Omega ; H)$ with $\mathbb{E}\left[\|\xi\|_{\infty}\right]<1$ and

$$
\Psi(0)=\Psi_{0}:=\mathbb{E}\left(\int_{\Omega} \xi \phi_{1} d x\right)>\zeta
$$

where $\zeta=\zeta(\lambda)$ is the largest root of the equation

$$
g(\tau):=\lambda f(\tau)-\lambda_{1} \tau=0 .
$$

Then the solution $u$ of (1.1)-(1.3) quenches in finite time. 
Proof. Following the same steps as in the proof of Theorem 5.4 we obtain that $\Psi(t)=$ $\mathbb{E}\left[\int_{\Omega} u \phi_{1} d x\right]$ satisfies the differential inequality

$$
\frac{d \Psi(t)}{d t} \geq-\lambda_{1} \Psi(t)+\lambda f(\Psi(t))=g(\Psi(t)), \quad t>0, \quad \Psi(0)=\Psi_{0} .
$$

Let $0<\zeta=\zeta(\lambda)<1$ be the largest root of the equation $g(\tau)=0$ then $g(\tau)>0$ for any $\tau>\zeta$; otherwise if $\zeta=0$ then $g(\tau)>0$ for any $0<\tau<1$.

Then by choosing $\Psi_{0}>\zeta$ we deduce

$$
t \leq \int_{\Psi_{0}}^{\Psi(t)} \frac{d \tau}{g(\tau)} \leq \int_{\Psi_{0}}^{1} \frac{d \tau}{g(\tau)}:=T^{*}<\infty .
$$

But the above relation guarantees that $\Psi(t)$ approaches 1 in finite time $T^{*}<\infty$. Therefore by virtue of (5.4) we derive that the solution of (1.1)-(1.3) quenches in finite time $T_{q} \leq$ $T^{*}$.

\section{REFERENCES}

[1] S. Assing Comparison of systems of stochastic partial differential equations, Stochastic Processes and their Applications 82 (1999), 259-282.

[2] P-L Chow, Stochastic Partial Differential Equations, Chapman and Hall/CRC, 2007.

[3] G. Da Prato \& J. Zabczyk, Stochastic Equations in Infinite Dimensions, Second Edition, Encyclopedia of Mathematics and its Applications vol. 152, Cambridge University Press, 2014.

[4] C. Donati-Martin \& N. Privault, White noise driven SPDEs with reflection, Probability Theory and Related Fields 95 (1993), 1-24.

[5] J. Duan \& W. Wang, Effective Dynamics of Stochastic Partial Differential Equations, Elsevier, USA, 2014.

[6] P. Esposito, N. Ghoussoub \& Y. Guo, Mathematical analysis of partial differential equations modeling electrostatic MEMS, Courant Lecture Notes in Mathematics, 20. Courant Institute of Mathematical Sciences, New York; American Mathematical Society, Providence, RI, 2010.

[7] G. Flores, G. Mercado, J.A. Pelesko, N. Smyth, Analysis of the dynamics and touchdown in a model of electrostatic MEMS, SIAM J. Appl. Math. 67 (2007) 434-446.

[8] N. Ghoussoub and Y. Guo, On the partial differential equations of electrostatic MEMS devices: stationary case, SIAM J. Math. Anal. 38 (2007), 1423-1449.

[9] N. Ghoussoub \& Y. Guo, On the partial differential equations of electrostatic MEMS devices II: dynamic case, Nonlinear Diff. Eqns. Appl. 15 (2008) 115-145.

[10] J.-S. GuO \& N.I. KaVAlLARIS, On a nonlocal parabolic problem arising in electromechanical MEMS control, Disc. Cont. Dynam. Systems 32 (2012) 1723-1746.

[11] Y. GUO, Z. PAN \& M.J. WARD, Touchdown and pull-in voltage behavior of a MEMS device with varying dielectric properties, SIAM J.Appl. Math. 166 (2006) 309-338.

[12] I. GyÖNGy \& C. RovirA, On $L^{p}$-solutions of semilinear stochastic partial differential equations Stochastic Process. Appl., 90(1) (2000), 83-108.

[13] K-M. HuI, The existence and dynamic properties of a parabolic nonlocal MEMS equation, Nonlinear Analysis: Theory, Methods \& Applications 74 (2011) 298-316.

[14] D.D. Joseph, T.S. Lundgren, Quasilinear Dirichlet problems driven by positive sources, Arch. Rat. Mech. Anal. 49 (1973) 241-269.

[15] S. Kaplan, On the growth of solutions of quasilinear parabolic equations, Comm. Pure Appl. Maths. 16 (1963) 327-330.

[16] N.I. Kavallaris, T. Miyasita \& T. Suzuki, Touchdown and related problems in electrostatic MEMS device equation, Nonlinear Diff. Eqns. Appl. 15 (2008) 363-385.

[17] N. I. Kavallaris, A. A. Lacey \& C. V. Nikolopoulos, On the quenching of a nonlocal parabolic problem arising in electrostatic MEMS control, Nonlinear Analysis: Theory, Methods \& Applications 138, (2016), 189-206, "Nonlinear Partial Differential Equations, in honor of Juan Luis Vàzquez for his 70th birthday". 
[18] G. J. Lord, C. E. Powell, and T. Shardlow, An Introduction to Computational Stochastic PDEs, Cambridge University Press, Cambridge, UK, 2014.

[19] J.A. Pelesko \& A.A. Triolo, Nonlocal problems in MEMS device control, J. Eng. Math. 41 (2001) 345-366.

[20] J.A. Pelesko \& D.H. Bernstein, Modeling MEMS and NEMS, Chapman Hall and CRC Press, 2002.

[21] M. Younis, MEMS Linear and Nonlinear Statics and Dynamics, Springer, New York, 2011.

Department of Mathematics, University of Chester, Thornton Science Park, Pool Lane, InCE, Chester CH2 4NU, UK

E-mail address: n.kavallaris@chester.ac.uk 